\title{
Analysis of Magnetization Currents
}

\author{
Neda Miteva ${ }^{\dagger}$ and Michael Slonim*
}

\begin{abstract}
An analytical method for harmonic estimation of magnetization currents has been developed. The method is based on the results of the waveforms qualitative analysis. The calculation and the experimental results are compared. A good matching is observed.
\end{abstract}

Keywords: Harmonics, Magnetization current, Hysteresis

\section{Introduction}

Transformers and coils with ferromagnetic cores are widely used in power systems and in power electronic converters. Magnetization currents of such elements are non-sinusoidal because of the hysteresis [1-4]. The hysteresis leads to the appearance of the harmonics in the transformer current. This phenomenon is widely discussed in current publications [5-7], emphasizing the importance of the problem.

The shape of the transformer magnetization current may be evaluated by numerical calculations as presented in $[5,6]$. In addition, the influence of the flux density on the harmonic current contents is discussed in [6]. The improved model of the transformer protection system is developed by changing the magnetization current spectrum [7]. All the research outlined above shows that the calculation of magnetization current harmonics is significant for engineering practice.

The present article proposes and applies an analytical approach based on experimental measurements. For this purpose, a qualitative analysis of experimentally obtained magnetization current shapes is carried out. The measured values of the magnetization current harmonics are compared with the calculated results.

\section{Description of the Measuring Procedure}

The magnetization currents of two transformers with ferromagnetic cores, $230 / 9 \mathrm{~V}$ and $230 / 24 \mathrm{~V}, 50 \mathrm{~Hz}$, are examined. The measurement set up is shown in Fig. 1. The voltage and current across the winding of no-load transformers have been measured by oscilloscope. The experimental results of the measurements appear in Fig. 2 and Fig. 3, where current and voltage waveforms are shown. The waveforms observed are quite similar. Such curves are typical for circuits with magnetic hysteresis $[1,2,5,6]$.

$\dagger$ Corresponding Author: Dept. of Electrical and Electronic Engineering, Ariel University of Samaria, Israel. (nedam@ariel.ac.il)

* Dept. of Electrical and Computer Engineering, Ben Gurion University, Israel. (michaels@bgu.ac.il)

Received: December 21, 2014; Accepted: August 11, 2016

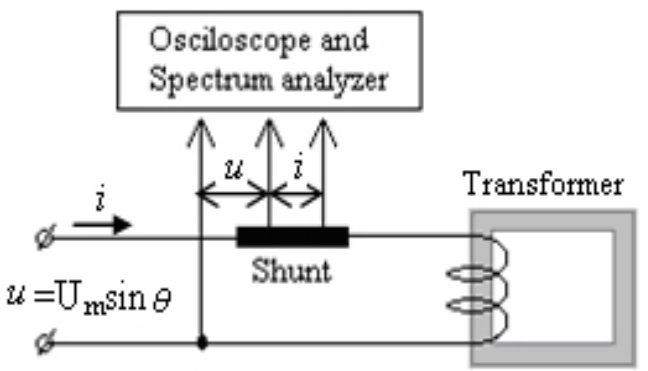

Fig. 1. Diagram of the measurement process

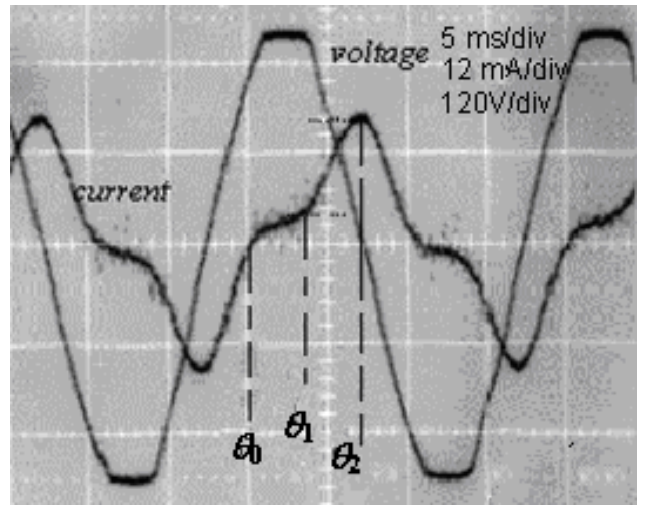

Fig. 2. Voltage and current waveform of $230 / 9 \mathrm{~V}$ transformer

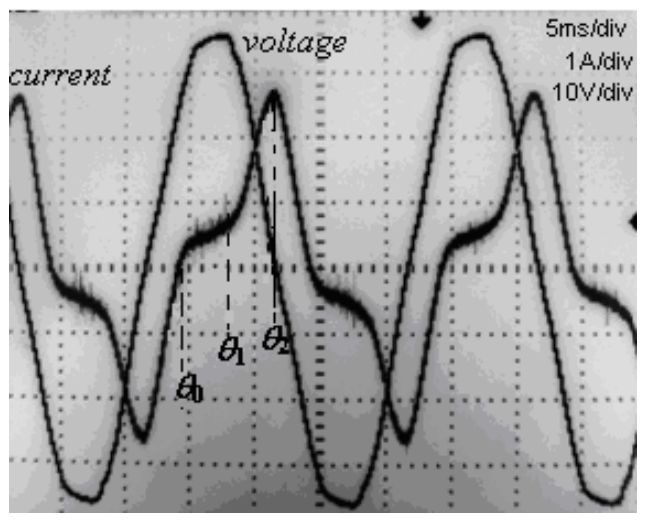

Fig. 3. Voltage and current waveform of $230 / 24 \mathrm{~V}$ transformer 


\section{Qualitative Analysis of the Magnetization Currents}

The harmonic contents of the currents have been measured by a spectrum analyzer. The results of the measurements are shown in Figs. 4 and 5, where the basic and third harmonics are the most essential. All the other harmonics (the fifth and higher) are very small and they will be neglected. Using these assumptions, the instantaneous magnetization current $i_{\mu}$ may be expressed as:

$$
i_{\mu}=i_{\mu 1}+i_{\mu 3}
$$

where $i_{\mu 1}$ and $i_{\mu 3}$ are the instantaneous currents of the basic and third harmonics, respectively.

There are three turning-points $\left(\theta_{0}, \theta_{1}\right.$ and $\left.\theta_{2}\right)$ of the current waveforms (Figs. 2 and 3 ). The currents equal zero at $\theta_{0}$, while they reach their maxima at the point $\theta_{2}$. The instant $\theta_{1}$ is the middle point of the interval $\theta_{0} \div \theta_{2}$. This is a turning point where the velocity of the currents' growth has changed.

The duration of the $\theta_{0} \div \theta_{2}$ interval is $120^{\circ}$ of the basic harmonic or a full period of the third harmonic. The intervals $\theta_{0} \div \theta_{1}$ and $\theta_{1} \div \theta_{2}$ are equal, i.e. $\theta_{1}-\theta_{0}=$

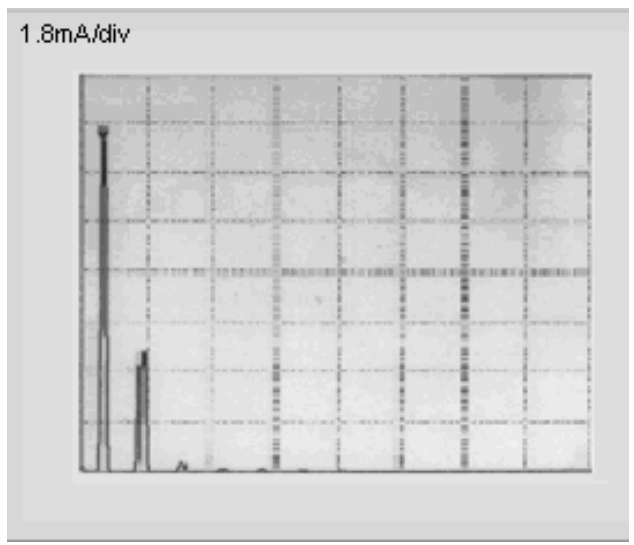

Fig. 4. Current harmonic contents of 230/9V transformer

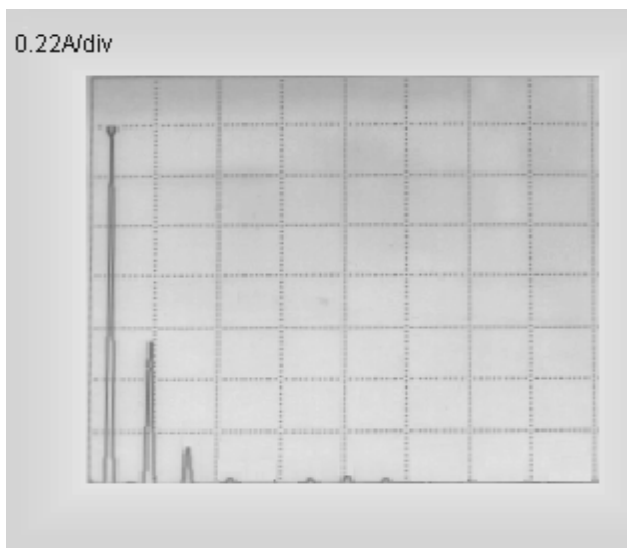

Fig. 5. Current harmonic contents of $230 / 24 \mathrm{~V}$ transformer $\theta_{2}-\theta_{1}=60^{\circ}$ of the basic $50 \mathrm{~Hz}$ frequency or $180^{\circ}$ of its third harmonic.

According to (1) the magnetization current may be decomposed as shown in Fig. 6, where the current itself is on the axis "a"; its basic harmonic is on the axis "b"; the third harmonic is on the axis "c". Using this decomposition, the following may be concluded. The current $i_{\mu}$ is zero at the moment $\theta_{0}$, i.e., at this moment, the basic and third harmonics are equal in their absolute values, but their signs are opposite (Fig. 6, b and c). During interval $\theta_{0} \div \theta_{1}$ the current $i_{\mu}$ grows slowly, while during interval $\theta_{1} \div \theta_{2}$ it grows rapidly. Such a behavior of the current $i_{\mu}$ may be explained assuming that during $\theta_{0} \div \theta_{2}$ interval the basic current harmonic grows, achieving a maximum around $\theta_{2}$. The third harmonic decreases from its maximum at $\theta_{0}$ to a minimum at $\theta_{1}$ and then it grows in the opposite direction during interval $\theta_{1} \div \theta_{2}$, achieving its maximum at the instant $\theta_{2}$.

Based on this analysis, the basic and third harmonics may be described as follows:

$$
\begin{gathered}
i_{\mu 1}(\theta)=\mathrm{I}_{m \mu 1} \sin \left(\theta-\theta_{0}-\psi_{1}\right), \\
i_{\mu 3}(\theta)=\mathrm{I}_{m \mu 3} \cos 3\left(\theta-\theta_{0}\right)
\end{gathered}
$$

where $\mathrm{I}_{m \mu 1}$ and $\mathrm{I}_{m \mu 3}$ are the amplitudes of the basic and third harmonics, respectively, and $\psi_{1}$ is the initial angle of the basic harmonics. Assuming that $\theta_{0}=0$ and substituting the Eqs. (2) and (3) in (1) the following current analytical expression is obtained:

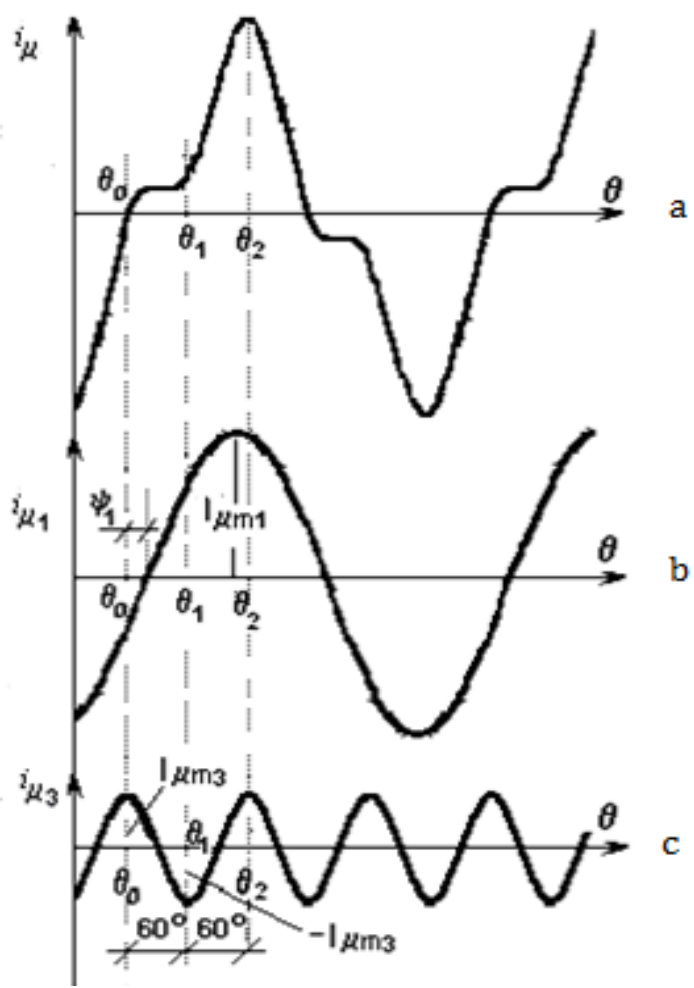

Fig. 6. Decomposition of the magnetization current 
Table 1. Measured and calculated current harmonics

\begin{tabular}{c|c|c|c|c}
\hline & & $\mathrm{I}_{\mathrm{m} \mu 1}$ & $\mathrm{I}_{\mathrm{m} \mu 3}$ & $\psi_{1}$ \\
\hline \multirow{4}{*}{ Fig. 2 } & $\mathrm{MR}(\mathrm{mA})$ & 11.9 & 4.26 & \\
\cline { 2 - 5 } & $\mathrm{CR}(\mathrm{mA})$ & 12 & 4.17 & $20.3^{\circ}$ \\
\cline { 2 - 5 } & $\varepsilon(\%)$ & 0.84 & 2.11 & \\
\hline \multirow{4}{*}{ Fig. 3 } & $\mathrm{MR}(\mathrm{A})$ & 1.59 & 0.64 & \\
\cline { 2 - 5 } & $\mathrm{CR}(\mathrm{A})$ & 1.66 & 0.61 & $21.6^{\circ}$ \\
\cline { 2 - 5 } & $\varepsilon(\%)$ & 4.4 & 4.67 & \\
\hline
\end{tabular}

$$
i_{\mu}(\theta)=\mathrm{I}_{m \mu 1} \sin \left(\theta-\psi_{1}\right)+\mathrm{I}_{m \mu 3} \cos 3 \theta
$$

The Eq. (4) contains three unknowns: $\mathrm{I}_{m \mu 1}, \mathrm{I}_{m \mu 3}$ and $\psi_{1}$. Their values may be calculated if a set of three algebraic equations containing these unknowns will be obtained. Measuring $i_{\mu}$ at $\theta=\theta_{0}=0, \theta=\theta_{1}=60^{\circ}$ and $\theta=\theta_{2}=120^{\circ}$ in Figs. 2 and 3 , a set of three algebraic equations will be obtained for the current of each transformer.

The first set of equations, corresponding to the current of the first transformer, is as follows:

$$
\begin{gathered}
i_{\mu}(0)=\mathrm{I}_{m \mu 1} \sin \left(-\psi_{1}\right)+\mathrm{I}_{m \mu 3}=0 \\
i_{\mu}(60)=\mathrm{I}_{m \mu 1} \sin \left(60-\psi_{1}\right)-\mathrm{I}_{m \mu 3}=3.5 \\
i_{\mu}(120)=\mathrm{I}_{m \mu 1} \sin \left(120-\psi_{1}\right)+\mathrm{I}_{m \mu 3}=16
\end{gathered}
$$

where all numerical values are measured in milli-amperes from Fig. 2.

Analogically, the second set of equations, corresponding to the second transformer, is as follows:

$$
\begin{aligned}
& i_{\mu}(0)=\mathrm{I}_{m \mu 1} \sin \left(-\psi_{1}\right)+\mathrm{I}_{m \mu 3}=0 \\
& i_{\mu}(60)=\mathrm{I}_{m \mu 1} \sin \left(60-\psi_{1}\right)-\mathrm{I}_{m \mu 3}=0.42 \\
& i_{\mu}(120)=\mathrm{I}_{m \mu 1} \sin \left(120-\psi_{1}\right)+\mathrm{I}_{m \mu 3}=2.25
\end{aligned}
$$

where all numerical values are measured in amperes from Fig. 3.

Solving the sets (5) and (6), the $\mathrm{I}_{m \mu 1}, \mathrm{I}_{m \mu 3}$ and $\psi_{1}$ values are calculated for each current. The calculated results (CR), the measured results (MR) and the calculated error $\mathcal{E}$ are shown in Table 1 .

The error $\varepsilon$ is found as follows:

$$
\varepsilon=\frac{|\mathrm{MR}-\mathrm{CR}|}{\mathrm{MR}} * 100, \%
$$

The observed errors are less than 5\% which proves that the proposed method is sound. The error of the phase angle $\psi_{1}$ is not calculated because the spectrum analyzer used did not allow its measurement.

\section{Conclusion}

An analytical method for harmonic calculation of magnetization currents is proposed. This method is based on qualitative analysis of the current waveforms, obtained experimentally. The magnetization currents of two unloaded transformers are analyzed. The currents' waveforms are similar and typical for the curves with hysteresis. Three characteristic points of the magnetization current are determined. These points are typical for the waveforms of the currents with hysteresis.

The qualitative analysis of the magnetization current waveforms allows developing the analytical method for calculation of the current basic and third harmonics. The results of the calculations are compared with the experimental results. A good matching is observed. The errors obtained are less than $5 \%$.

\section{References}

[1] Abu Hasem Chowdhury, William Grady and Ewald Fuchs, "An investigation of the harmonic characteristics of transformer excitation current under nonsinusoidal supply voltage", IEEE Trans. on Power Delivery, vol. 14, No. 2, pp. 450-458, April 1999.

[2] Irving Morris Gottlieb, Practical Transformer Handbook, Newnes, 1998, pp. 6-8, 77-102.

[3] Silvano Cincotti, Michele Marshesi, Antonino Serri, "A neural network model of parametric nonlinear hysteretic inductors", IEEE Trans. on Magnetics, vol. 34, Issue 5, pp. 3040-3043, Sept. 1998.

[4] Byung-Il Know, Seong-Jin Park, Seung-Chan Park "Forward converter analysis by the method of coupling electromagnetic field with hysteresis and circuit equations", IEEE Trans. on Magnetics, vol. 36, Issue 4, pp. 1426-1430, July 2000.

[5] Antônio Flavio Licarião Nogueira, Gabriel Grunitzki Facchinello and Leonardo Adriano Ramos, "Prediction of magnetizing currents in power transformers using numerically simulated open-circuit tests", IJRRAS, vol. 17, Issue 2, pp. 141-149, Nov. 2013.

[6] Ismail Daut, Syafruddin Hasan and Soib Taib, "Magnetizing Current, Harmonic Content and Power Factor as the Indicators of Transformer Core Saturation", Journal of Clean Energy Technologies, Vol. 1, No. 4, Oct. 2013.

[7] Seyed Ali Mousavi, Claes Carrander and Göran Engdahl, "Comprehensive Study on Magnetization Current Harmonics of Power Transformers due to GICs", International Conference on Power Systems Transients (IPST2013), Vancouver, Canada, July 1820, 2013. 


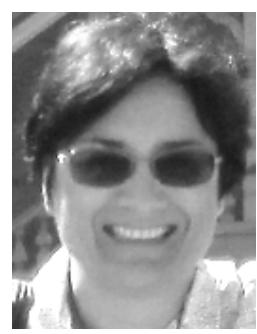

Neda Miteva She received an M. Sc. degree in electrical engineering from the Higher Institute for Machine and Electrical Engineering, Sofia, Bulgaria in 1986. She received a Ph.D. degree in electrical engineering from Tel Aviv University in 2003. Since 2003 she has been working at the Ariel University of Samaria, Israel. Her specific field of interest is estimation of active power and calculation of current harmonics in nonlinear electrical circuit elements.

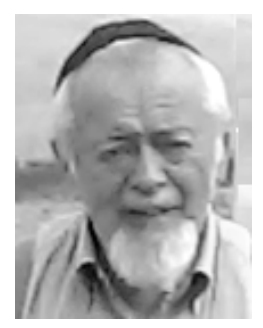

Michael Slonim $\mathrm{He}$ has graduated Leningrad Polytechnic Institute in 1959 and has received an M. Sc. degree in electrical engineering (High Voltage Technic). He has received a Ph.D. degree from the same Institute in 1966 in electrical engineering transient process analysis). He is Senior Scientist of Academy of Sciences (former USSR) from 1971. He has been with Ben Gurion University, Beer Sheva, Israel from 1972 to 2007 (to-day he is Professor Emeritus). He is Member of Israeli Independent Academy for Development of Sciences from 2008. 